\title{
COHOMOLOGICALLY KÄHLER MANIFOLDS WITH NO KÄHLER METRICS
}

\author{
MARISA FERNÁNDEZ, VICENTE MUÑOZ, and JOSÉ A. SANTISTEBAN
}

\author{
Received 20 November 2002
}

\begin{abstract}
We show some examples of compact symplectic solvmanifolds, of dimension greater than four, which are cohomologically Kähler and do not admit Kähler metric since their fundamental groups cannot be the fundamental group of any compact Kähler manifold. Some of the examples that we study were considered by Benson and Gordon (1990). However, whether such manifolds have Kähler metrics was an open question. The formality and the hard Lefschetz property are studied for the symplectic submanifolds constructed by Auroux (1997) and some consequences are discussed.
\end{abstract}

2000 Mathematics Subject Classification: 53D35, 57R17, 55P62.

1. Introduction. A symplectic manifold $(M, \omega)$ is a pair consisting of a $2 n$ dimensional differentiable manifold $M$ together with a closed 2-form $\omega$ which is nondegenerate (i.e., $\omega^{n}$ never vanishes). The form $\omega$ is called symplectic. By the Darboux theorem, in canonical coordinates, $\omega$ can be expressed as

$$
\omega=\sum_{i=1}^{n} d x^{i} \wedge d x^{n+i} .
$$

Any symplectic manifold $(M, \omega)$ carries an almost complex structure $J$ compatible with the symplectic form $\omega$, which means that $\omega(X, Y)=\omega(J X, J Y)$ for any $X, Y$ vector fields on $M$ (see $[22,23])$. If $(M, \omega)$ has an integrable almost complex structure $J$ compatible with the symplectic form $\omega$ such that the Riemannian metric $g$, given by $g(X, Y)=-\omega(J X, Y)$, is positive definite, then $(M, \omega, J)$ is said to be a Kähler manifold with Kähler metric $g$.

The problem of how compact symplectic manifolds differ topologically from Kähler manifolds led, during the last years, to the introduction of several geometric methods for constructing symplectic manifolds (see [5, 8, 15, 20, 21]). The symplectic manifolds presented there do not admit a Kähler metric since they are not formal or do not satisfy hard Lefschetz theorem, or they fail both properties of compact Kähler manifolds.

The purpose of this paper is to show that the formality and the hard Lefschetz property of any compact symplectic manifold $M$ are not sufficient conditions to imply the existence of a Kähler metric on $M$. We describe three 
families of compact symplectic solvmanifolds $M^{6}(c), P^{6}(c)$, and $N^{6}(c)$ of dimension 6 , and a family of compact symplectic solvmanifolds $N^{8}(c)$ of dimension 8, each of which is formal and satisfies the hard Lefschetz property. Thus, they are cohomologically Kähler, their odd Betti numbers are even (see [19]), and their even Betti numbers are nonzero.

In [13], there are given examples of 4-dimensional compact symplectic manifolds which are cohomologically Kähler but do not possess complex structures, so they admit no Kähler metrics. This is done by appealing to classification theorems of Kodaira and Yau that are specific to complex dimension 2.

In our case, we resort, in Section 3, to the properties of the fundamental group of a compact Kähler manifold given by Campana [7] to show that none of the manifolds $M^{6}(c), N^{6}(c), P^{6}(c)$, and $N^{8}(c)$ admit Kähler metrics (see Theorems 3.3 and 3.5). A similar technique was used in [14] to prove the existence of 4-dimensional Donaldson symplectic submanifolds with no complex structures. The manifolds $N^{6}(c)$ as well as the manifolds $P^{6}(c)$ were considered in [6]. There, Benson and Gordon show that they are cohomologically Kähler. However, whether or not they have a Kähler metric was an open question.

On the other hand, in Section 4, we study the formality and the hard Lefschetz property for the symplectic submanifolds obtained by Auroux in [3] as an extension to higher-rank bundles of the symplectic submanifolds constructed by Donaldson in [11]. Let $(M, \omega)$ be a compact symplectic manifold of dimension $2 n$ with $[\omega] \in H^{2}(M)$ having a lift to an integral cohomology class, and let $E$ be any Hermitian vector bundle over $M$ of rank $r$. In [3], Auroux proved the existence of some integer number $k_{0}$ such that for any $k \geq k_{0}$, there is a symplectic submanifold $Z_{r} \hookrightarrow M$ of dimension $2(n-r)$ whose homology class realizes the Poincaré dual of $k^{r}[\omega]^{r}+k^{r-1} c_{1}(E)[\omega]^{r-1}+\cdots+c_{r}(E)$, where $c_{i}(E)$ denotes the $i$ th Chern class of the vector bundle $E$. For such manifolds the inclusion $j: Z_{r} \hookrightarrow M$ induces on cohomology:

(i) an isomorphism $j^{*}: H^{i}(M) \rightarrow H^{i}\left(Z_{r}\right)$ for $i<n-r$;

(ii) a monomorphism $j^{*}: H^{i}(M) \hookrightarrow H^{i}\left(Z_{r}\right)$ for $i=n-r$.

As a consequence of this study, we get some examples of Auroux symplectic submanifolds (in particular, nonparallelizable manifolds) of dimension 6 which are formal and hard Lefschetz, but do not carry Kähler metrics.

2. Formal manifolds. First, we need some definitions and results about minimal models. Let $(A, d)$ be a differential algebra, that is, $A$ is a graded commutative algebra over the real numbers, with a differential $d$ which is a derivation, that is, $d(a \cdot b)=(d a) \cdot b+(-1)^{\operatorname{deg}(a)} a \cdot(d b)$, where $\operatorname{deg}(a)$ is the degree of $a$.

A differential algebra $(A, d)$ is said to be minimal if

(i) $A$ is free as an algebra, that is, $A$ is the free algebra $\wedge V$ over a graded vector space $V=\oplus V^{i}$,

(ii) there exists a collection of generators $\left\{a_{\tau}, \tau \in I\right\}$, for some well-ordered index set $I$, such that $\operatorname{deg}\left(a_{\mu}\right) \leq \operatorname{deg}\left(a_{\tau}\right)$ if $\mu<\tau$ and each $d a_{\tau}$ is 
expressed in terms of preceding $a_{\mu}(\mu<\tau)$. This implies that $d a_{\tau}$ does not have a linear part, that is, it lives in $\wedge V^{>0} \cdot \wedge V^{>0} \subset \wedge V$.

Morphisms between differential algebras are required to be degree-preserving algebra maps which commute with the differentials. Given a differential algebra $(A, d)$, we denote by $H^{*}(A)$ its cohomology. We say that $A$ is connected if $H^{0}(A)=\mathbb{R}$, and $A$ is one-connected if, in addition, $H^{1}(A)=0$.

We will say that $(\mathcal{M}, d)$ is a minimal model of the differential algebra $(A, d)$ if $(M, d)$ is minimal and there exists a morphism of differential graded algebras $\rho:(\mathcal{M}, d) \rightarrow(A, d)$ inducing an isomorphism $\rho^{*}: H^{*}(\mathcal{M}) \rightarrow H^{*}(A)$ on cohomology. Halperin [17] proved that any connected differential algebra $(A, d)$ has a minimal model unique up to isomorphism.

A minimal model $(\mu, d)$ is said to be formal if there is a morphism of differential algebras $\psi:(\mathcal{M}, d) \rightarrow\left(H^{*}(\mathcal{M}), d=0\right)$ that induces the identity on cohomology. The formality of a minimal model can be distinguished as follows.

THEOrem 2.1 (see [10]). A minimal model $(M, d)$ is formal if and only if $M=\wedge V$ and the space $V$ decomposes as a direct sum $V=C \oplus N$ with $d(C)=0$, $d$ is injective on $N$ and such that every closed element in the ideal $I(N)$ generated by $N$ in $\bigwedge V$ is exact.

A minimal model of a connected differentiable manifold $M$ is a minimal model $(\bigwedge V, d)$ for the de Rham complex $(\Omega M, d)$ of differential forms on $M$. If $M$ is a simply connected manifold, the dual of the real homotopy vector space $\pi_{i}(M) \otimes \mathbb{R}$ is isomorphic to $V^{i}$ for any $i$. We will say that $M$ is formal if its minimal model is formal or, equivalently, the differential algebras $(\Omega M, d)$ and $\left(H^{*}(M), d=0\right)$ have the same minimal model. (For details see, for example, $[10,16])$

In [14], the condition of formal manifold is weaken to $s$-formal manifold as follows.

DEFINITION 2.2. Let $(\mathcal{M}, d)$ be a minimal model of a differentiable manifold $M$. We say that $(\mathcal{M}, d)$ is $s$-formal, or $M$ is an $s$-formal manifold $(s \geq 0)$ if $M=\bigwedge V$ such that for each $i \leq s$, the space $V^{i}$ of generators of degree $i$ decomposes as a direct sum $V^{i}=C^{i} \oplus N^{i}$, where the spaces $C^{i}$ and $N^{i}$ satisfy the three following conditions:

(i) $d\left(C^{i}\right)=0$,

(ii) the differential map $d: N^{i} \rightarrow \wedge V$ is injective,

(iii) any closed element in the ideal $I_{s}=I_{s}\left(\bigoplus_{i \leq s} N^{i}\right)$, generated by $\bigoplus_{i \leq s} N^{i}$ in $\bigwedge\left(\oplus_{i \leq s} V^{i}\right)$, is exact in $\bigwedge V$.

The relation between the formality and the $s$-formality for a manifold is given in the following theorem.

THEOREM 2.3 (see [14]). Let $M$ be a connected and orientable compact differentiable manifold of dimension $2 n$ or $(2 n-1)$. Then $M$ is formal if and only if it is $(n-1)$-formal. 


\section{Formal and hard Lefschetz symplectic manifolds with no Kähler metric.} In this section, we show the existence of compact symplectic manifolds of dimension greater than 4, which do not admit Kähler metrics even when they are formal and hard Lefschetz.

EXAMPLE 3.1 (the manifolds $M^{6}(c)$ [9]). Let $G(c)$ be the connected completely solvable Lie group of dimension 5 consisting of matrices of the form

$$
a=\left(\begin{array}{cccccc}
e^{c z} & 0 & 0 & 0 & 0 & x_{1} \\
0 & e^{-c z} & 0 & 0 & 0 & y_{1} \\
0 & 0 & e^{c z} & 0 & 0 & x_{2} \\
0 & 0 & 0 & e^{-c z} & 0 & y_{2} \\
0 & 0 & 0 & 0 & 1 & z \\
0 & 0 & 0 & 0 & 0 & 1
\end{array}\right)
$$

where $x_{i}, y_{i}, z \in \mathbb{R}(i=1,2)$ and $c$ is a nonzero real number. Then a global system of coordinates $x_{1}, y_{1}, x_{2}, y_{2}$, and $z$ for $G(c)$ is given by $x_{i}(a)=x_{i}$, $y_{i}(a)=y_{i}$, and $z(a)=z$. A standard calculation shows that a basis for the right invariant 1-forms on $G(c)$ consists of

$$
\left\{d x_{1}-c x_{1} d z, d y_{1}+c y_{1} d z, d x_{2}-c x_{2} d z, d y_{2}+c y_{2} d z, d z\right\}
$$

Alternatively, the Lie group $G(c)$ may be described as a semidirect product $G(c)=\mathbb{R} \ltimes_{\psi} \mathbb{R}^{4}$, where $\psi(z)$ is the linear transformation of $\mathbb{R}^{4}$ given by the matrix

$$
\left(\begin{array}{cccc}
e^{c z} & 0 & 0 & 0 \\
0 & e^{-c z} & 0 & 0 \\
0 & 0 & e^{c z} & 0 \\
0 & 0 & 0 & e^{-c z}
\end{array}\right)
$$

for any $z \in \mathbb{R}$. Thus, $G(c)$ has a discrete subgroup $\Gamma(c)=\mathbb{Z} \ltimes_{\psi} \mathbb{Z}^{4}$ such that the quotient space $\Gamma(c) \backslash G(c)$ is compact. Therefore, the forms $d x_{i}-c x_{i} d z$, $d y_{i}+c y_{i} d z$, and $d z(i=1,2)$ descend to 1 -forms $\alpha_{i}, \beta_{i}$, and $\gamma(i=1,2)$ on $\Gamma(c) \backslash G(c)$.

Now, we consider the manifold $M^{6}(c)=\Gamma(c) \backslash G(c) \times S^{1}$. Hence, there are 1 -forms $\alpha_{1}, \beta_{1}, \alpha_{2}, \beta_{2}, \gamma$, and $\eta$ on $M^{6}(c)$ such that

$$
d \alpha_{i}=-c \alpha_{i} \wedge \gamma, \quad d \beta_{i}=c \beta_{i} \wedge \gamma, \quad d \gamma=d \eta=0,
$$

where $i=1,2$, and such that at each point of $M^{6}(c),\left\{\alpha_{1}, \beta_{1}, \alpha_{2}, \beta_{2}, \gamma, \eta\right\}$ is a basis for the 1 -forms on $M^{6}(c)$. Using Hattori's theorem [18], we compute the 
real cohomology of $M^{6}(c)$ :

$$
\begin{aligned}
& H^{0}\left(M^{6}(c)\right)=\langle 1\rangle, \\
& H^{1}\left(M^{6}(c)\right)=\langle[\gamma],[\eta]\rangle, \\
& H^{2}\left(M^{6}(c)\right)=\left\langle\left[\alpha_{1} \wedge \beta_{1}\right],\left[\alpha_{1} \wedge \beta_{2}\right],\left[\alpha_{2} \wedge \beta_{1}\right],\left[\alpha_{2} \wedge \beta_{2}\right],[\gamma \wedge \eta]\right\rangle, \\
& H^{3}\left(M^{6}(c)\right)=\left\langle\left[\alpha_{1} \wedge \beta_{1} \wedge \gamma\right],\left[\alpha_{1} \wedge \beta_{2} \wedge \gamma\right],\left[\alpha_{2} \wedge \beta_{1} \wedge \gamma\right],\left[\alpha_{2} \wedge \beta_{2} \wedge \gamma\right],\right. \\
& {\left.\left[\alpha_{1} \wedge \beta_{1} \wedge \eta\right],\left[\alpha_{1} \wedge \beta_{2} \wedge \eta\right],\left[\alpha_{2} \wedge \beta_{1} \wedge \eta\right],\left[\alpha_{2} \wedge \beta_{2} \wedge \eta\right]\right\rangle, } \\
& H^{4}\left(M^{6}(c)\right)=\left\langle\left[\alpha_{1} \wedge \beta_{1} \wedge \alpha_{2} \wedge \beta_{2}\right],\left[\alpha_{1} \wedge \beta_{1} \wedge \gamma \wedge \eta\right],\left[\alpha_{1} \wedge \beta_{2} \wedge \gamma \wedge \eta\right],\right. \\
& {\left.\left[\alpha_{2} \wedge \beta_{1} \wedge \gamma \wedge \eta\right],\left[\alpha_{2} \wedge \beta_{2} \wedge \gamma \wedge \eta\right]\right\rangle, } \\
& H^{5}\left(M^{6}(c)\right)=\left\langle\left[\alpha_{1} \wedge \beta_{1} \wedge \alpha_{2} \wedge \beta_{2} \wedge \gamma\right],\left[\alpha_{1} \wedge \beta_{1} \wedge \alpha_{2} \wedge \beta_{2} \wedge \eta\right]\right\rangle, \\
& H^{6}\left(M^{6}(c)\right)=\left\langle\left[\alpha_{1} \wedge \beta_{1} \wedge \alpha_{2} \wedge \beta_{2} \wedge \gamma \wedge \eta\right]\right\rangle .
\end{aligned}
$$

Therefore, the Betti numbers of $M^{6}(c)$ are

$$
\begin{aligned}
& b_{0}\left(M^{6}(c)\right)=b_{6}\left(M^{6}(c)\right)=1, \\
& b_{1}\left(M^{6}(c)\right)=b_{5}\left(M^{6}(c)\right)=2, \\
& b_{2}\left(M^{6}(c)\right)=b_{4}\left(M^{6}(c)\right)=5, \\
& b_{3}\left(M^{6}(c)\right)=8 .
\end{aligned}
$$

Proposition 3.2. The manifold $M^{6}(c)$ is 2-formal and so formal. Moreover, $M^{6}(c)$ has a symplectic form $\omega$ such that $\left(M^{6}(c), \omega\right)$ satisfies the hard Lefschetz property.

Proof. To prove that $M^{6}(c)$ is 2 -formal, we see that its minimal model must be a differential graded algebra $(\mu, d), \mathcal{M}$ is the free algebra of the form $M=\bigwedge\left(a_{1}, a_{2}\right) \otimes \bigwedge\left(b_{1}, b_{2}, b_{3}, b_{4}\right) \otimes \wedge V^{\geq 3}$ where the generators $a_{i}$ have degree 1 , the generators $b_{j}$ have degree 2 , and the differential $d$ is given by $d a_{i}=$ $d b_{j}=0$, where $i=1,2$ and $1 \leq j \leq 4$. The morphism $\rho: \mathcal{M} \rightarrow \Omega(M)$, inducing an isomorphism on cohomology, is defined by $\rho\left(a_{1}\right)=\gamma, \rho\left(a_{2}\right)=\eta, \rho\left(b_{1}\right)=$ $\alpha_{1} \wedge \beta_{1}, \rho\left(b_{2}\right)=\alpha_{1} \wedge \beta_{2}, \rho\left(b_{3}\right)=\alpha_{2} \wedge \beta_{1}$, and $\rho\left(b_{4}\right)=\alpha_{2} \wedge \beta_{2}$.

According to Definition 2.2, we get $C^{1}=\left\langle a_{1}, a_{2}\right\rangle$ and $N^{1}=0$, thus $M^{6}(c)$ is 1-formal. Moreover, $M^{6}(c)$ is 2-formal since $C^{2}=\left\langle b_{1}, b_{2}, b_{3}, b_{4}\right\rangle$ and $N^{2}=0$. Now, the formality of $M^{6}(c)$ follows from Theorem 2.3.

We define the symplectic form $\omega$ on $M^{6}(c)$ by

$$
\omega=\alpha_{1} \wedge \beta_{1}+\alpha_{2} \wedge \beta_{2}+\gamma \wedge \eta
$$

Then, the maps $[\omega]: H^{2}\left(M^{6}(c)\right) \rightarrow H^{4}\left(M^{6}(c)\right)$ and $[\omega]^{2}: H^{1}\left(M^{c}(k)\right) \rightarrow$ $H^{5}\left(M^{6}(c)\right)$ are isomorphisms. Thus, $\left(M^{6}(c), \omega\right)$ satisfies the hard Lefschetz property. 
The manifolds $M^{6}(c)$ were considered in [9]. There, the formality of $M^{6}(c)$ is obtained as a consequence of the existence of a morphism $\left(H^{*}\left(M^{6}(c)\right), d=\right.$ $0) \rightarrow\left(\Omega^{*}\left(M^{6}(c)\right), d\right)$ that induces the identity on cohomology. Such a morphism is defined by linearity choosing closed forms representatives for each cohomology class. However, whether or not $M^{6}(c)$ has a Kähler metric was an open question.

THEOREM 3.3. The manifold $M^{6}(c)$ does not admit Kähler metrics.

Proof. In order to show that $M^{6}(c)$ does not admit Kähler metric, notice that $\Gamma=\pi_{1}\left(M^{6}(c)\right)$ is a product $\Gamma=\Gamma(c) \times \mathbb{Z}$. Moreover, its abelianization is $H_{1}\left(M^{6}(c) ; \mathbb{Z}\right)$, and thus, it has rank 2 . We will see that $\Gamma$ cannot be the fundamental group of any compact Kähler manifold.

The exact sequence

$$
0 \longrightarrow \mathbb{Z}^{4} \longrightarrow \Gamma \longrightarrow \mathbb{Z}^{2} \longrightarrow 0
$$

shows that $\Gamma$ is solvable of class 2 , that is, $D^{3} \Gamma=0$. Moreover, its rank is 6 by additivity (see [1] for details).

Assume now that $\Gamma=\pi_{1}(X)$, where $X$ is a compact Kähler manifold. According to Arapura-Nori's theorem (see [2, Theorem 3.3]), there exists a chain of normal subgroups

$$
0=D^{3} \Gamma \subset Q \subset P \subset \Gamma,
$$

such that $Q$ is torsion, $P / Q$ is nilpotent, and $\Gamma / P$ is finite. The exact sequence (3.8) implies that $\Gamma$ has no torsion, and so $Q=0$. As $\Gamma / P$ is torsion, thus finite, we have $\operatorname{rank} P=\operatorname{rank} \Gamma=6$. Now, the finite inclusion $P \subset \Gamma$ defines a finite cover $p: Y \rightarrow X$ that is also compact Kähler and it has fundamental group $P$.

We show that $P$ cannot be the fundamental group of any compact Kähler manifold. For this, we use Campana's result (see [7, Corollary 3.8, page 313]) that states that if $G$ is the fundamental group of a Kähler manifold such that $G$ is nilpotent and non-abelian, then $G$ has rank greater than or equal to 9.

Since $P$ is the fundamental group of the Kähler manifold $Y, P$ is nilpotent, it has rank less than 9 , and it has to be abelian. This is impossible since any pair of nonzero elements $e \in \mathbb{Z}^{2} \subset \Gamma=\mathbb{Z}^{2} \ltimes \mathbb{Z}^{4}, f \in \mathbb{Z}^{4} \subset \Gamma$ do not commute (see, e.g., [12, page 22]).

EXAMPLE 3.4 (the manifolds $N^{6}(c)$ ). We consider the connected completely solvable Lie group $G(c)$ of dimension 3 consisting of matrices of the form

$$
a=\left(\begin{array}{cccc}
e^{c z} & 0 & 0 & x \\
0 & e^{-c z} & 0 & y \\
0 & 0 & 1 & z \\
0 & 0 & 0 & 1
\end{array}\right)
$$


where $x, y, z \in \mathbb{R}(i=1,2)$ and $c$ is a nonzero real number. Then a global system of coordinates $x, y$, and $z$ for $G(c)$ is given by $x(a)=x, y(a)=y$, and $z=z$. A standard calculation shows that a basis for the right invariant 1-forms on $G(c)$ consists of

$$
\{d x-c x d z, d y+c y d z, d z\}
$$

Let $\Gamma(c)$ be a discrete subgroup of $G(c)$ such that the quotient space Sol(3)= $\Gamma(c) \backslash G(c)$ is compact (for the existence of such a subgroup $\Gamma(c)$ see [4, page 20]). Hence, the forms $d x-c x d z, d y+c y d z$, and $d z$ all descend to 1-forms $\alpha, \beta$, and $\gamma$ on $\operatorname{Sol}(3)$ such that

$$
d \alpha=-c \alpha \wedge \gamma, \quad d \beta=c \beta \wedge \gamma, \quad d \gamma=0 .
$$

We use again Hattori's theorem [18] to compute the real cohomology of Sol(3)

$$
\begin{aligned}
& H^{0}(\operatorname{Sol}(3))=\langle 1\rangle, \\
& H^{1}(\operatorname{Sol}(3))=\langle[\gamma]\rangle, \\
& H^{2}(\operatorname{Sol}(3))=\langle[\alpha \wedge \gamma]\rangle, \\
& H^{3}(\operatorname{Sol}(3))=\langle[\alpha \wedge \beta \wedge \gamma]\rangle .
\end{aligned}
$$

Denote by $M^{4}(c)$ the product $M^{4}(c)=\operatorname{Sol}(3) \times S^{1}$. In [13], it is proved that $M^{4}(c)$ is cohomologically Kähler (in fact, it has the same minimal model as $T^{2} \times S^{2}$ ) and it does not carry complex structures, and so it carries no Kähler metrics. This is done by appealing to classification theorems of Kodaira and Yau that are specific to complex surfaces.

Next, we consider other examples in dimensions 6 and 8 related also with Sol(3). Define the manifolds $N^{6}(c)=\operatorname{Sol}(3) \times \operatorname{Sol}(3), P^{6}(c)=\operatorname{Sol}(3) \times T^{3}$, and $N^{8}(c)=\operatorname{Sol}(3) \times \operatorname{Sol}(3) \times T^{2}=N^{6}(c) \times T^{2}$. These manifolds are formal since they are product of formal manifolds.

From the definition of $N^{6}(c)$ and from (3.12), one can check that there are 1 -forms $\alpha_{1}, \beta_{1}, \gamma_{1}, \alpha_{2}, \beta_{2}$, and $\gamma_{2}$ on $N^{6}(c)$ such that

$$
d \alpha_{i}=-c \alpha_{i} \wedge \gamma_{i}, \quad d \beta_{i}=c \beta_{i} \wedge \gamma_{i}, \quad d \gamma_{i}=0,
$$

where $i=1,2$, and such that at each point of $N^{6}(c),\left\{\alpha_{1}, \beta_{1}, \gamma_{1}, \alpha_{2}, \beta_{2}, \gamma_{2}\right\}$ is a basis for the 1 -forms on $N^{6}(c)$. We define the symplectic form $\omega_{1}$ on $N^{6}(c)$ by

$$
\omega_{1}=\alpha_{1} \wedge \beta_{1}+\alpha_{2} \wedge \beta_{2}+\gamma_{1} \wedge \gamma_{2}
$$

We use again (3.12) to show that there is a basis $\left\{\alpha_{1}, \beta_{1}, \gamma_{1}, \eta_{1}, \eta_{2}, \eta_{3}\right\}$ for the 1-forms on $P^{6}(c)$ such that

$$
d \alpha_{1}=-c \alpha_{1} \wedge \gamma_{1}, \quad d \beta_{1}=c \beta_{1} \wedge \gamma_{i}, \quad d \gamma_{1}=d \eta_{j}=0,
$$


for $1 \leq j \leq 3$, since $P^{6}(c)=\operatorname{Sol}(3) \times T^{3}$. Thus, the 2 -form $\omega_{2}$, defined by

$$
\omega_{2}=\alpha_{1} \wedge \beta_{1}+\gamma_{1} \wedge \eta_{1}+\eta_{2} \wedge \eta_{3}
$$

is a symplectic form on $P^{6}(c)$.

It is clear that $N^{8}(c)$ is a symplectic manifold since it is the product of symplectic manifolds. In fact, a symplectic form $\omega_{3}$ on $N^{8}(c)$ is given by

$$
\omega_{3}=\omega_{1}+\eta
$$

where $\eta$ is a symplectic form on the 2-torus $T^{2}$.

One can check that the manifolds $N^{6}(c), P^{6}(c)$, and $N^{8}(c)$ are cohomologically Kähler. Now, using an argument similar to the one given in Theorem 3.3, we get the following theorem.

THEOREM 3.5. The manifolds $N^{6}(c), P^{6}(c)$, and $N^{8}(c)$ are formal and hard Lefschetz but they admit no Kähler metrics.

We notice that the manifolds $N^{6}(c)$ and $P^{6}(c)$ were considered as examples of cohomologically Kähler manifolds by Benson and Gordon in [6]. However, whether or not they have a Kähler metric was an open question.

\section{Formality and hard Lefschetz property for Auroux symplectic submani-}

folds. In this section, we study the conditions under which Auroux symplectic manifolds are formal and/or satisfy the hard Lefschetz theorem.

Let $(M, \omega)$ be a compact symplectic manifold of dimension $2 n$ with $[\omega] \in$ $H^{2}(M)$ admitting a lift to an integral cohomology class, and let $E$ be any Hermitian vector bundle over $M$ of rank $r$. In [3], Auroux constructs symplectic submanifolds $Z_{r} \hookrightarrow M$ of dimension $2(n-r)$ whose Poincaré dual is $\operatorname{PD}\left[Z_{r}\right]=k^{r}[\omega]^{r}+k^{r-1} c_{1}(E)[\omega]^{r-1}+\cdots+c_{r}(E)$ for any integer number $k$ large enough, where $c_{i}(E)$ denotes the $i$ th Chern class of the vector bundle E. Moreover, these submanifolds satisfy a Lefschetz theorem in hyperplane sections, meaning that the inclusion $j: Z_{r} \hookrightarrow M$ is $(n-r)$-connected, that is, the map there $j^{*}: H^{i}(M) \rightarrow H^{i}\left(Z_{r}\right)$ is an isomorphism for $i<n-r$ and a monomorphism for $i=n-r$.

In general, let $X$ and $Y$ be compact manifolds. We say that a differentiable map $f: X \rightarrow Y$ is a homotopy $s$-equivalence $(s \geq 0)$ if it induces isomorphisms $f^{*}: H^{i}(Y) \stackrel{\cong}{\rightrightarrows} H^{i}(X)$ on cohomology for $i<s$, and a monomorphism $f^{*}$ : $H^{s}(Y) \hookrightarrow H^{s}(X)$ for $i=s$. Therefore, for any Auroux symplectic submanifold, the inclusion $j: Z_{r} \hookrightarrow M$ is a homotopy $(n-r)$-equivalence.

THEOREM 4.1 (see [14]). Let $X$ and $Y$ be compact manifolds and let $f: X \rightarrow Y$ be a homotopy s-equivalence. If $Y$ is $(s-1)$-formal, then $X$ is $(s-1)$-formal.

As a consequence of Theorem 4.1, we get the following corollary. 
COROLlary 4.2. Let $M$ be a compact symplectic manifold of dimension $2 n$ and let $Z_{r} \hookrightarrow M$ be an Auroux submanifold of dimension 2 $(n-r)$. For each $s \leq(n-r-1)$, if $M$ is $s$-formal then $Z_{r}$ is $s$-formal. In particular, $Z_{r}$ is formal if $M$ is $(n-r-1)$-formal.

In order to continue the analysis of the Auroux symplectic submanifolds we introduce the following definition.

DEFINITION 4.3. Let $(M, \omega)$ be a compact symplectic manifold of dimension $2 n$. We say that $M$ is $s$-Lefschetz with $s \leq(n-1)$ if

$$
[\omega]^{n-i}: H^{i}(M) \longrightarrow H^{2 n-i}(M)
$$

is an isomorphism for all $i \leq s$. By extension, if we say that $M$ is $s$-Lefschetz with $s \geq n$, then we just mean that $M$ is hard Lefschetz.

THEOREM 4.4. Let $(M, \omega)$ be a compact symplectic manifold of dimension $2 n$ such that the de Rham cohomology class $[\omega] \in H^{2}(M)$ has a lift to an integral cohomology class, and let $Z_{r} \rightarrow M$ be an Auroux submanifold of dimension $2(n-$ $r)$. Then, for large enough $k$ and for each $s \leq(n-r-1)$, if $M$ is $s$-Lefschetz, then $Z_{r}$ is $s$-Lefschetz. Therefore, $Z_{r}$ is hard Lefschetz if $M$ is $(n-r-1)$-Lefschetz.

Proof. From now on, we denote by $L$ the complex line bundle over $M$ whose first Chern class is $c_{1}(L)=[\omega]$. Let $p=2(n-r)-i$, where $i \leq(n-r-1)$, and we consider the map $j^{*}: H^{p}(M) \rightarrow H^{p}\left(Z_{r}\right)$ induced by the inclusion $j$ on cohomology. First, we claim that for $[z] \in H^{p}(M)$ it holds that

$$
j^{*}[z]=0 \Longleftrightarrow[z] \cup c_{r}\left(E \otimes L^{\otimes k}\right)=0,
$$

for large values of the parameter $k$. This can be shown via Poincaré duality. Clearly, $j^{*}[z]=0$ if and only if $j^{*}[z] \cdot a=0$ for any $a \in H^{i}\left(Z_{r}\right)$. Since there is an isomorphism $H^{i}\left(Z_{r}\right) \cong H^{i}(M)$ for $i \leq(n-r-1)$, we can assume that there exists a closed $i$-form $x$ on $M$ with $\left[\left.x\right|_{Z_{r}}\right]=[\hat{x}]=a, \hat{x}$ being the differential form on $Z_{r}$ given by $\hat{x}=j^{*}(x)$. So

$$
j^{*}[z] \cdot[\hat{x}]=\int_{Z} \hat{z} \wedge \hat{x}=\int_{M} z \wedge x \wedge \tilde{c}_{r}\left(E \otimes L^{\otimes k}\right)
$$

since $\left[Z_{r}\right]=\operatorname{PD}\left[c_{r}\left(E \otimes L^{\otimes k}\right)\right]$, where $\tilde{c}_{r}\left(E \otimes L^{\otimes k}\right)$ is a differential form on $M$ representing $c_{r}\left(E \otimes L^{\otimes k}\right)$. Hence, $j^{*}[z]=0$ if and only if $\left([z] \cup c_{r}\left(E \otimes L^{\otimes k}\right)\right) \cup$ $[x]=0$ for all $[x] \in H^{i}(M)$, from where the claim follows.

Now, consider an arbitrary norm on $H^{*}(M)$; for example, the $L^{2}$-norm on harmonic forms. Let $S \subset H^{i}(M)$ be the unitary sphere, and denote by $K$ an upper bound of

$$
\left\|\left\{a \cup[\omega]^{n-i-q} \cup c_{q}(E) \mid a \in S, q=1, \ldots, r\right\}\right\| .
$$


On the other hand, the $s$-Lefschetz property of $M$ implies that $S \cup[\omega]^{n-i} \subset$ $H^{2 n-i}(M)$ does not contain zero. Therefore, there is a lower bound $K^{\prime}>0$ for the set

$$
\left\|\left\{a \cup[\omega]^{n-i} \mid a \in S\right\}\right\|
$$

Now, for any $[z] \in S$, we obtain

$$
[z] \cup[\omega]^{n-r-i} \cup\left(k^{r}[\omega]^{r}+k^{r-1}[\omega]^{r-1} \cup c_{1}(E)+\cdots+c_{r}(E)\right) \neq 0
$$

taking $k>(r-1) K / K^{\prime}$. Thus, $\hat{z} \cup\left[\hat{\omega}^{n-r-i}\right] \neq 0$ for any $[\hat{z}] \in H^{i}\left(Z_{r}\right)$, which proves that $Z_{r}$ is also $s$-Lefschetz.

We now consider the compact symplectic solvmanifolds $N^{8}(c)$ defined in Example 3.4. Since $N^{8}(c)$ has a symplectic form that defines an integral cohomology class, there exist Auroux symplectic submanifolds $Z_{r} \hookrightarrow N^{8}(c)$ of dimension $2(4-r)$ for $1 \leq r \leq 3$.

Proposition 4.5. Any Auroux symplectic submanifold $Z_{r} \hookrightarrow N^{8}(c)$ is formal and hard Lefschetz. Moreover, $Z_{r}$ does not admit Kähler metrics for $r=1,2$, and the submanifolds $Z_{3} \hookrightarrow N^{8}(c)$ are Kähler.

Proof. From Theorem 3.5, Corollary 4.2, and Theorem 4.4, we get that any Auroux symplectic submanifold $Z_{r} \leftrightarrow N^{8}(c)$ is formal and hard Lefschetz. Moreover, a similar argument to the one given in Theorem 3.3 proves that the submanifolds $Z_{r}$ do not admit Kähler metrics for $r=1,2$.

AcknowLEdgments. We are grateful to the referee for valuable suggestions and comments. This work has been partially supported by grants MCYT (Spain) Projects BFM2000-0024 and BFM2001-3778-C03-02. Also partially supported by the European Contract Human Potential Programme, Research Training Network HPRN-CT-2000-00101.

\section{REFERENCES}

[1] J. Amorós, M. Burger, K. Corlette, D. Kotschick, and D. Toledo, Fundamental Groups of Compact Kähler Manifolds, Mathematical Surveys and Monographs, vol. 44, American Mathematical Society, Rhode Island, 1996.

[2] D. Arapura and M. Nori, Solvable fundamental groups of algebraic varieties and Kähler manifolds, Compositio Math. 116 (1999), no. 2, 173-188.

[3] D. Auroux, Asymptotically holomorphic families of symplectic submanifolds, Geom. Funct. Anal. 7 (1997), no. 6, 971-995.

[4] L. Auslander, L. Green, and F. Hahn, Flows on Homogeneous Spaces, Annals of Mathematics Studies, no. 53, Princeton University Press, New Jersey, 1963.

[5] C. Benson and C. S. Gordon, Kähler and symplectic structures on nilmanifolds, Topology 27 (1988), no. 4, 513-518.

[6] _ Kähler structures on compact solvmanifolds, Proc. Amer. Math. Soc. 108 (1990), no. 4, 971-980. 
[7] F. Campana, Remarques sur les groupes de Kähler nilpotents [Remarks on nilpotent Kähler groups], Ann. Sci. École Norm. Sup. (4) 28 (1995), no. 3, 307-316 (French).

[8] L. A. Cordero, M. Fernández, and A. Gray, Symplectic manifolds with no Kähler structure, Topology 25 (1986), no. 3, 375-380.

[9] L. C. de Andrés, M. Fernández, M. de León, and J. J. Mencía, Some six-dimensional compact symplectic and complex solvmanifolds, Rend. Mat. Appl. (7) 12 (1992), no. 1, 59-67.

[10] P. Deligne, P. A. Griffiths, J. Morgan, and D. Sullivan, Real homotopy theory of Kähler manifolds, Invent. Math. 29 (1975), no. 3, 245-274.

[11] S. K. Donaldson, Symplectic submanifolds and almost-complex geometry, J. Differential Geom. 44 (1996), no. 4, 666-705.

[12] M. Fernández, M. de León, and M. Saralegui, A six-dimensional compact symplectic solvmanifold without Kähler structures, Osaka J. Math. 33 (1996), no. 1, 1935.

[13] M. Fernández and A. Gray, Compact symplectic solvmanifolds not admitting complex structures, Geom. Dedicata 34 (1990), no. 3, 295-299.

[14] M. Fernández and V. Muñoz, On the formality and the hard Lefschetz property for Donaldson symplectic manifolds, preprint, 2002, http://arXiv.org/ abs/math.SG/0211017.

[15] R. E. Gompf, A new construction of symplectic manifolds, Ann. of Math. (2) 142 (1995), no. 3, 527-595.

[16] P. A. Griffiths and J. W. Morgan, Rational Homotopy Theory and Differential Forms, Progress in Mathematics, vol. 16, Birkhäuser Boston, Massachusetts, 1981.

[17] S. Halperin, Lectures on minimal models, Mém. Soc. Math. Fr. (N.S.) (1983), no. 910, 261.

[18] A. Hattori, Spectral sequence in the de Rham cohomology of fibre bundles, J. Fac. Sci. Univ. Tokyo Sect. IA Math. 8 (1960), 289-331.

[19] O. Mathieu, Harmonic cohomology classes of symplectic manifolds, Comment. Math. Helv. 70 (1995), no. 1, 1-9.

[20] D. McDuff, Examples of simply-connected symplectic non-Kählerian manifolds, J. Differential Geom. 20 (1984), no. 1, 267-277.

[21] W. P. Thurston, Some simple examples of symplectic manifolds, Proc. Amer. Math. Soc. 55 (1976), no. 2, 467-468.

[22] A. Tralle and J. Oprea, Symplectic Manifolds with no Kähler Structure, Lecture Notes in Mathematics, vol. 1661, Springer-Verlag, Berlin, 1997.

[23] A. Weinstein, Lectures on Symplectic Manifolds, Regional Conference Series in Mathematics, no. 29, American Mathematical Society, Rhode Island, 1977.

Marisa Fernández: Departamento de Matemáticas, Facultad de Ciencias, Universidad del País Vasco, Apartado 644, 48080 Bilbao, Spain

E-mail address: mtpfero1@1g.ehu.es

Vicente Muñoz: Departamento de Matemáticas, Facultad de Ciencias, Universidad Autónoma de Madrid, 28049 Madrid, Spain

E-mail address: vicente.munoz@uam.es

José A. Santisteban: Departamento de Matemáticas, Facultad de Ciencias, Universidad del País Vasco, Apartado 644, 48080 Bilbao, Spain

E-mail address: mtpsae1j@1g.ehu.es 


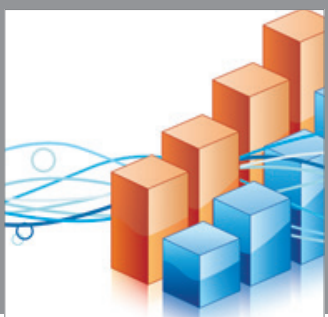

Advances in

Operations Research

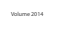

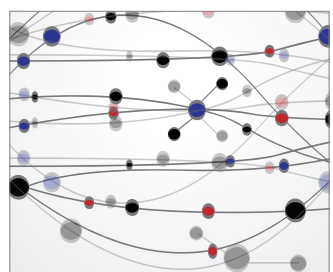

\section{The Scientific} World Journal
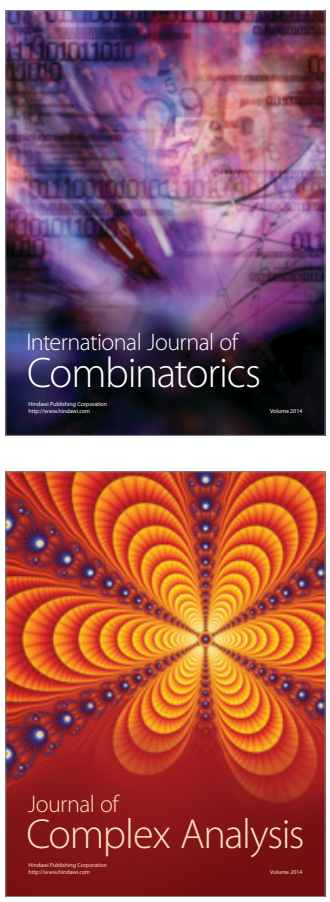

International Journal of

Mathematics and

Mathematical

Sciences
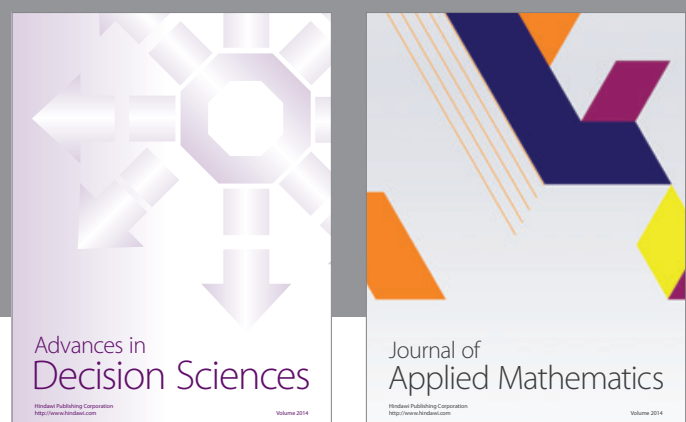

Journal of

Applied Mathematics
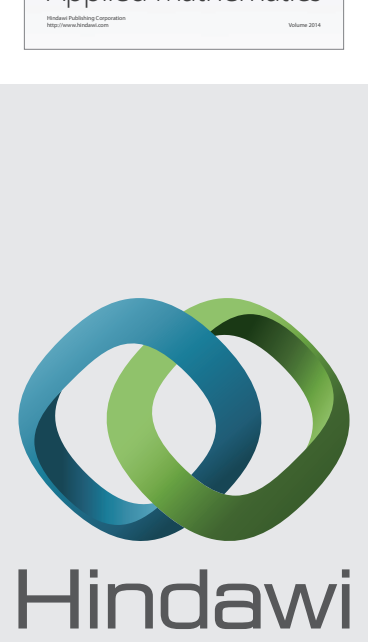

Submit your manuscripts at http://www.hindawi.com
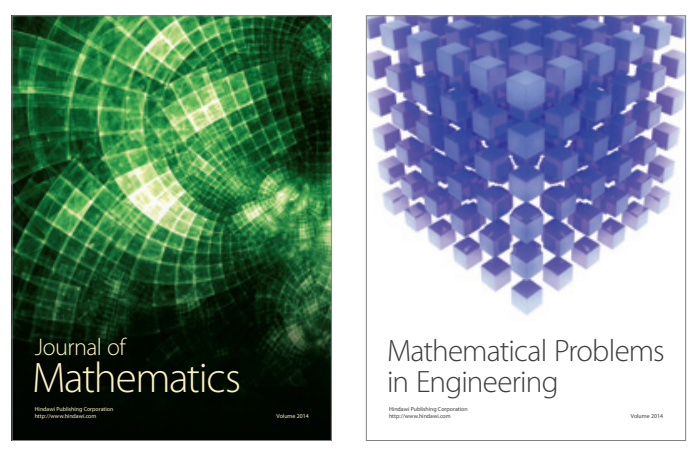

Mathematical Problems in Engineering
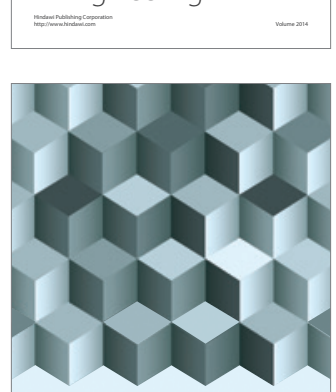

Journal of

Function Spaces
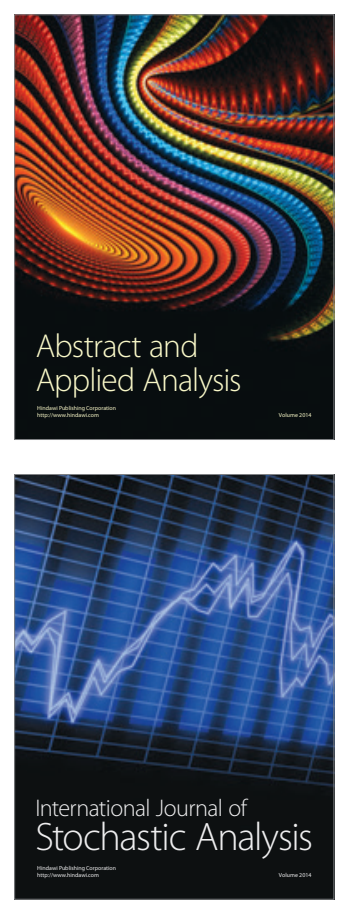

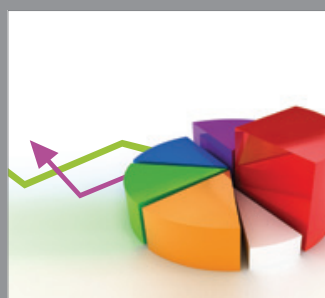

ournal of

Probability and Statistics

Promensencen
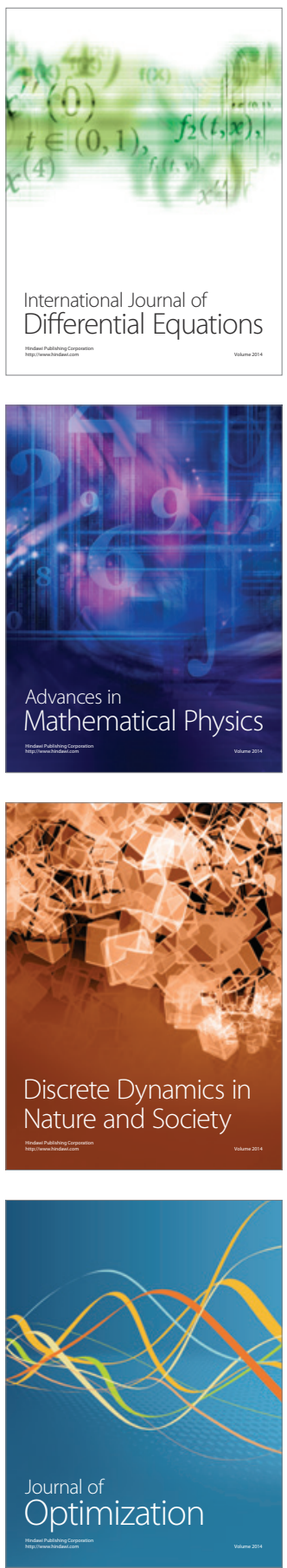